tion as existed in Case I, as regards the ocular and maxim in medicine," says Dr. Austin Flint, "that the nasal mucous membrane, except a slight degree of therapeutical indications derived from science and pannus, which would account for the great loss in vision.

This patient was under treatment for two months, when she was dismissed as cured. She recently informed me by letter that her eyes have remained well, that vision has improved, and she is now able to read ordinary print.

The above cases were both treated, prior to my treatment, by eminent oculists, from nine to twelve months each, without perceptible improvement; one had spent a term at Eureka Springs, Ark. Other cases could be reported, but it is unnecessary; these show, by the immediate and perfect recovery in each case, the efficacy and correctness of the treatment. The conclusions, therefore, as the above cases had persistently resisted the usual method of treatment for like conditions, and the rapid and perfect recovery from the treatment as above described, is positive evidence of the correctness of the diagnosis and value of treatment.

The local sedative treatment to the nasal mucous membrane relieved the irritation reflex of the conjunctiva, thus making recovery possible, which otherwise could not be, while the primary point of disease was disregarded.

\section{THE RATIONAL TREATMENT OF PULMONARY DISEASES BY THE PNEUMATIC CABINET.}

BY W. EVERETT SMITH, M.D. (HARV.)

FELLOW OF THE MASSACHUSETTS MEUICAL SOCIETY ; MEMHER OF THE AMERICAN MEDICAL ASSOCIATION; FORMERLY ASST, HHYSICIAN

TO THE MASSACHUSETTS HOME FOR INTEMPERATE WOMEN.

The use of the Pneumatic Cabinet ${ }^{1}$ marks a new era in the treatment of lung diseases. Until within about thirty years, pulmonary consumption was almost universally regarded as an incurable disease. Indeed, it was only the occasional discovery of fibrous or cicatricial tissue and of calcareous masses in the lungs of patients who had died of other diseases, and in whose lungs there were no evidences of any recent inflammatory processes, that gradually led the medical world to believe that consumption could ever be cured. Yet still the fact remained that the vast majority of patients with destructive lung diseases died. The possibility of a cure being, however, conclusively established, the causes of these diseases began to be more carefully studied, with the hope of finally discovering some treatment directed to the removal of these causes.

Many have been the methods advised - the internal administration of wood naphtha, cod-liver oil, phosphate of lime, the hypophosphites, chlorate of potassa, benzoate of soda, various modes of counter-irritation, mercurialization, antimonial and other nauseating cough mixtures, vacuum cures, inhalations, etc., etc. All may have their modicum of value, but all have failed to work the desired cure because they were not founded upon a physiological basis. "It is a sound

\footnotetext{
Introduced to the profession by Dr. Herb. F. Williams, of Brooklyn, N. Y. See article in New York Medical Record, Jan. 17, 1885.
}

from nature, as a rule, should harmonize, and I will add that the true principles of therapeutics are in accordance with the dictates of common sense."

Adequately, to cope with disease, then, it is necessary thoroughly to understand the functions and requirements of health; and it is suggestive of the value of the treatment of lung diseases by the Pneumatic Cabinet that "its development is in line with and depends upon physiological axioms. The life of lung tissue depends upon pure air; any interruption or irregularity in this supply means lung disease. Whenever, then, lung tissue is consolidated, as in the early stages of tubercular phthisis, or as the result of pleuritic effusions or empyema, or by direct inflammation, as in an unresolved pneumonia, the life and healthful activity of the respiratory system is seriously impeded. To expand these consolidated portions, and at the same time to deposit remedial and antiseptic agents in the inflamed lung cells, is the method and the theory of the treatment by the Pneumatic Cabinet.

The Cabinet is practically an air-tight iron safe about seven feet high, two feet wide and two and a half feet deep; large enough, in fact, for a patient to stanci or sit in. In the rear is a heavy door fitted with bolts, but ingeniously opened and closed by a single turn of the hand. In front is a large plate glass window, through which passes a gutta percha tube having a stop-cock on the outside, and ending in a trumpet shape for receiving the medicated spray. The tube within the Cabinet is fitted to a flexible rubber mouthpiece for the patient. The patient enters the Cabinet and seats himself opposite the window. The door is closed and the air is rarefied by an air pump to the desired degree as represented by the fall of mercury in a barometer connected with the inside of the Cabinet. 'The degree of rarefaction is usually that represented by a barometric fall of from one-tenth of an inch to an inch and a half, each tenth of an.inch being equivalent to an altitude of Ioo feet as regards the removal of external pressure from the chest walls. The patient now inserts the inhaling tube in his mouth and compresses the nostrils with the fingers to prevent the escape of air through the nose; the stop-cock is opened and the outside air rushes in, carrying with it the medicated spray, which is atomized by a powerful cylinder of compressed air. The effect is a forced involuntary inspiration followed by a forced voluntary expiration. These respiratory movements are continued for several minutes, but upon the least fatigue the stop-cock is closed, the patient removes the tube from the mouth, and breathes the rarefied air of the Cabinet until ready for another application. The treatment varies in duration from ten to thirty minutes, and is repeated either daily or at intervals of two or three days, the number of applications varying, the greatest number being 105-a case of acute catarrhal phthisis in Dr. Williams' practice, which recovered.

The peculiar value of this treatment lies "in the combination of the medicated spray with the increased strength of the inspiratory movements." A much 
fuller expansion of the chest is produced than is possible by an ordinary full inspiration, and at the same time the medicated spray being carried to the lung cells with much greater force than by a natural inspiration, is deposited in the very deepest and most remote portions of the lungs. Antiseptic air thus admin-. istered, must condense under the increased pressure due to the active expiratory effort, and be deposited in cavities or other diseased portions of the lungs.

At the same time one must not neglect to consider the beneficial effects of removing the external pressure of the atmosphere from the chest walls. In the first place, the active and passive movements of the respiratory act being reversed, the breathing takes place not merely with the ordinary tidal volume of air (which is carried by inspiration only to the trachea and larger bronchial tubes, reaching the lung cells in obedience simply to the law of the diffusion of gases), but also with the much larger volume of complemental air. When properly conducted therefore, a calisthenic action is produced which expands and develops even the healthy chest, and which is beneficial from a mere hygienic standpoint. Indeed, spirometric tests before and after a course of treatment, have demonstrated an increase in vital or volumetric capacity of from twenty-five to one hundred per cent., and a corre sponding development both of chest measurements and of chest expansion. The removal of the external pressure causes moreover such an expansion of air in the bronchial tubes as forcibly to expel secretions, which may have so accumulated as seriously to impede the proper expansion of air vesicles. Finally, the influence of this treatment upon the circulation is very instructive and worthy of the most careful study. We know that in ordinary inspiration, arterial tension is increased while in expiration it is correspondingly decreased. In forced respiration, in a rarefied atmosphere, the proportionate difference between the blood pressure in inspiration and in expiration is increased, the main arterial pressure is increased, but the capillary blood pressure in the lung tissue is so far decreased as actually to decrease the liability to pulmonary hæmorrhage.

This modification of the capillary blood pressure can scarcely be other than beneficial to any inflammatory process in the lung tissue. The danger, how ever, should not be overlooked that with fatty hearts, aneurismal dilatations, atheromatous arteries and denuded arteries lying in cavities of the lungs, the most serious or even fatal injury might be done by a careless or ill-advised use of the Cabinet. This, however, is no argument against the value of the treatment; it is, in fact, a direct argument in favor of its power over lung expansion.

The principal agents thus far used in the spray have been the bichloride of mercury, iodine, carbolic acid and phenyl. "While the evidence," says Dr. Williams, "is strongly presumptive that the use of these germicides diminished the quantity of bacilli in several instances and obliterated them in others, the opportunity for following out this line of inquiry has not furnished sufficient data to permit an unqualified statement." To kill bacteria is one thing, to kill germs is another; and all evidence seems to point to the fact that it is impossiple to keep germs out of the body. What then can inhalations do?

Several years ago, Prof. Tyndall made some interesting studies on this subject, and discovered that although by a continuous boiling he could not sterilize solutions, he could sterilize them by many successive boilings. Although he might not at once destroy the fully-developed bacteria, he could destroy their offspring while they were too young to produce a fresh generation. It is true that this may be only a theory, but it is a theory which will I think stand the test of experience and it is the theory underlying the treatment by antiseptic inhalations.

'The materials which have been found most useful as antiseptic inhalations are not mere gases which are mingled with the inspired air and then pass out with it, but they are vapors of soluble bodies which are deposited upon lung tissue. Upon every moist bronchial tube, upon the walls of cavities and around, if not in, congested areas the inhaled vapor is condensed to render the soil barren for germ growth. "For if the difference between a soil fit and one unfit for bacterial growth is to be measured, for example, by the difference between the functional activity and resulting vital resistance of an upper and a lower lobe of the lung, surely it is not unreasonable to hope that by impregnating the pulmonary tissues with antiseptic material which we know renders them less fitted for the cultivation of bacilli, we may be able to make up the lacking resistance and so prevent the access of disease." 1

For a further confirmation of the proposition that by discontinuous antiseptic inhalations bacilli can be made to disappear from the sputa, the reader is referred to a very interesting paper read by Dr. V. Y. Bowditch before the Suffolk District Medical Society, Nov. I I, 1885 .

The effect of these inhalations in the Cabinet upon the cough depends upon the lesion in the lung. In nearly every case reported by Dr. Williams, and in all of the cases that I have been privileged to see, the cough and expectoration have been much reduced, there has been an increase of appetite and of weight, a tendency to a more restful sleep and a reduction of the fever. Indeed it can be safely claimed that where fever is due to the absorption of pus from a lung cavity accessible to the air, the introduction of an antiseptic will as certainly reduce the fever as will the washing of a uterus in puerperal septicæmia, or the cleansing of any suppurating wound. The cases that have thus far been reported have certainly given proof of the possibilities of recovery from serious lung lesions. Of three cases of unresolved pneumonia, Dr. Williams reports all cured; of seven cases of primary infiltration, six cured and one improved; of nine cases of acute catarrhal phthisis, four cured and one improved, while of nine cases of chronic fibrous phthisis, eight showed marked improvement.

The danger when looking at such a history of treatment is, that inconsiderate enthusiasm may prevent one from fairly weighing evidence, which must in all justness be impartially considered. It is our duty, however, as conscientious and progressive physicians,

'Solomon G. Smith, M.D., British Medical Journal, Feb. 23, 1884. 
to investigate the subject carefully and without prejudice, patiently developing what is good in the method, and gradually learning what is its proper place in the healing art.

Thus far its greatest value seems to have been in the treatment of early stages of phthisis, in fibroid phthisis, in chronic bronchitis, in emphysematous conditions of the lungs, and in the consolidations resulting from pleurisy, empyema, and unresolved pneumonia. Upon the Continent of Europe, the applications of compressed air have proved of inestimable value in the treatment of asthma, and it is not improbable that similar successes may soon be recorded here. lo meet these various indications, an ingenious mechanism attached to the Cabinet permits the operator at will either to rarefy the air within the ( $a b$ inet to a given degree, or to compress it to a given degree, or alternately to rarefy and compress it synchronously with the respiratory act.

Our only method of determining the merits of the treatment is by a careful study and a complete record of all cases. I trust to be able soon to report some original investigations upon the subject, studying it in its relations to and effect upon the bealthy organism as well as the diseased.

I94 West Brookline St., Boston, Mass.

\section{RUPTURE OF THE RECTUM, AND PROLAPSE OF THE SMALL INTESTINE. ${ }^{1}$}

BY S. B. LYON, M.D.,

GOVERNMENT HOSPITAL FOR THE INSANE, WASHINGTON, D, C.

I desire to present some notes on an unusual case of rupture of the rectum, and protrusion of a loop of the small intestine through the opening in the wall, and from the anus.

The patient was a woman of 5o, suffering with melancholia of a very active type, accompanied with delusions largely relating to the pelvic organs; she often fancied herself pregnant, or being delivered; and her conversation, which was almost continuous during waking hours, was on subjects forbidden in polite society. She had a remarkable talent for making remarks exceedingly embarrassing to both the physician and the nurses. One result of her delusive ideas of her pregnant condition, was a disposition at times to assist nature by introducing her hand into her vagina or rectum, and pulling or working at those organs, so that a tendency to prolapsus of both uterus and rectum existed, and these conditions had been reduced more than once. The disposition to interfere dangerously with herself made constant watchfulness and some restraint necessary.

A few days since, when visiting the ward, the nurse called my attention to a mass of viscera which had descended from the anus, and an examination showed at once that it was not a question of a prolapsus of the rectum, such as had previously occurred, but that the protruding mass was made up of a part of the smail intestine, and the appearance of omentum and the serous surface of the gut excluded a mere turning inside out. Only a rupture of the large intestine

IRead before the Medical Society of the District of Columbia, October 14,1885 . and the protrusion through the opening thus made of the small gut, could explain the condition. Th: rectum, with its internal and external sphincters, was in its nornial condition, not even slightly prolapsed by the weight and tension of the protruding mass.

Ordinary modes of reduction were attempted, but with no success; the weakened condition of the woman from shock, and long-continued exhausting excitement, precluded surgical interference. Within forty-eight hours death resulted without any sthenic symptoms. The following is Dr. Blackburn's report of the autopsy:

"Mrs. opening the abcomen a peritonitis of the folds of intestine in the polvic region was revealed. The peritoneal surfaces in that region were agglutinated by recent lymph, and a moderate quantity of reddish serum with floating lymph was contained in the cavity. On further examination a rupture of the anterior wall of the rectum within the recto-uterine pouch (Douglas's) was discovered. Through this opening a large loop of the ileum, with its mesentery, had passed. No escape of faces had occurred, as the intestine was adherent to the edges of the rupture. The portion of the intestine protruded began about six inches from the ileococal valve, and was about eighteen inches in length. The loop was discolored and covered with lymph. Some strangulation had occurred, shown by the thickening of the intestine and the discoloration of the part. Examination of the vagina and os uteri revealed an old laceration of the os, and erosion of the posterior lip of the cervix."

The only mention I have seen of a similar condition is under the head of hernia, as "Hedrocele," in Gross's "Surgery," where he quotes Dr. Uhde, of Brunswick; but no sack existing in the present case, it is doubtful if it is described under "Hedrocele."

\section{MEDICAL PROGRESS.}

\section{MATERIA MEDICA AND THERAPEUTICS.}

The Hippurates of IJime AND OF Jithitim.Dr. V. Poulet considers the hippurate of lime as the best preparation of calcareous salts for use in the system, and as especially useful in affections of the urinary passages; in affections of the liver; in diseases of the skin and mucous membrane, where they are dependent upon functional vices of the liver or upon lymphatism; in a great number of diseases of the alimentary canal, both of the stomach and intestine; in diabetes; and in chronic rheumatism, gout, etc.

In urinary affections, the subacute cystitis of the neck of the bladder yields satisfactorily to its use, with relief to the frequent desire to urinate, pain on urinating, etc. In certain cases where the urine manifests an abnormal alkaline reaction it becomes promptly acid again by the use of this drug. In every case, there is a return to the normal limpidity and a ressation of the presence of the mucous globules. It is quite as useful in urinary lithiasis from whatever cause, phosphaturia, uraturia, or oxaluria. If the theory of the 